\title{
Time-resolved observation of coherent phonons by the Franz-Keldysh effect
}

\author{
G. C. Cho, H. J. Bakker, T. Dekorsy, and H. Kurz \\ Institut für Halbleitertechnik II, Rheinisch-Westfälische Technische Hochschule Aachen, \\ Sommerfeldstrasse 24, D-52056 Aachen, Germany
}

\begin{abstract}
We report on the time-resolved observation of coherent optical phonons in GaAs by the Franz-Keldysh effect. This effect leads to a modulation of the optical interband transitions with a nonlinear dependence on the macroscopic electric field associated with the coherent phonons. We find that the nonlinear electro-optic effect forms a far more efficient detection mechanism for coherent phonons than the linear electro-optic effect near the band gap.
\end{abstract}

In recent years, the study of coherent phenomena with ultrashort laser pulses has become a matter of growing interest. The use of subpicosecond laser pulses allows a direct observation of coherent vibrations in molecules, ${ }^{1}$ phonons in solid-state materials, ${ }^{2-4}$ and electronic wave-packet oscillations in quantum-well structures in the time domain. ${ }^{5}$ In previous studies the excitation mechanisms and the dephasing of coherent oscillations have been addressed.

Recent investigations of coherent phonons in solid-state materials have shown that the excitation results from the impulsive interaction of the material with laser pulses whose durations are shorter than the period of the oscillation. In molecules, coherent vibrations can be excited by impulsivestimulated Raman scattering processes. ${ }^{1}$ In narrow-band-gap semiconductors, the strong interband excitation of carriers leads to the excitation of coherent phonons by the impulsive elongation of the bonds. ${ }^{3}$ It was found that in these materials the breathing mode of the coherent lattice vibrations modifies the band gap via the deformation potential. ${ }^{6}$ In III-V semiconductors, the femtosecond excitation of free carriers results in the impulsive screening of the surface depletion field due to an ultrafast charge separation. This mechanism generates coherent longitudinal optical (LO) phonons, which are associated with an oscillating macroscopic electric field. ${ }^{2,7}$ These field changes have been observed in timeresolved reflectivity measurements via the linear electrooptic effect. $^{2}$

In principle, the reflectivity changes induced by coherent phonons in III-V semiconductors arise from the modulation of the optical susceptibility at the phonon frequency, which is expressed by the coupling of the Raman tensor with the coherent motion of the atomic displacement. In a first-order approximation the phonon-induced reflectivity changes $\Delta R$ can be described by

$$
\Delta R \sim(\partial \chi / \partial Q) \Delta Q=\left[(\partial \chi / \partial Q)_{F}+(\partial \chi / \partial F)_{Q}(\partial F / \partial Q)\right] \Delta Q
$$

where $\chi$ is the real part of the optical susceptibility, $Q$ the atomic displacement, and $F$ the macroscopic electric field. The first and second terms at the right-hand side in Eq. (1) are the deformation potential and the electro-optic contribution, respectively. This first-order Raman tensor determines the usual allowed Raman scattering with its specific polarization selection rules. 8,9
However, in the vicinity of critical points in the electronic band structure, nonlinear effects such as forbidden Raman scattering and electric-field-induced Raman scattering by LO phonons become strongly enhanced. ${ }^{8,9}$ The polarization selection rule for these types of Raman tensors is different from that of allowed Raman scattering. Both effects are based on the modulation of the optical susceptibility through the nonlinear electro-optic (NEO) contribution to the Raman tensor. The resonance enhancement of the electric-fieldinduced Raman scattering at the $E_{0}$ band gap is determined by the Franz-Keldysh effect, ${ }^{8}$ which can be described by the third-order nonlinear optical susceptibility $\chi^{(3)}$ in a low-field approximation. ${ }^{10}$

Here, we demonstrate the modification of the optical transition at a critical point $\left(E_{0}\right)$ in GaAs induced by coherent LO phonons as detected via the time-resolved Franz-Keldysh effect. The Franz-Keldysh enhancement is isotropic, e.g., it does not depend on the polarization of the detecting laser light. Thus, it can be easily separated from the linear electrooptic (LEO) effect, for which the polarization selection rule is the same as that of the first-order Raman tensor of Eq. (1). We observe a strong spectral dependence of the NEO effects in the vicinity of the $E_{0}$ band gap of GaAs driven by coherent phonons.

Our experiments are performed with a fast-scan pumpprobe setup, which is equipped with a shaker scanner instead of a stepper motor for realizing the pump-probe time delay. This real-time data acquisition without using a chopper for the pump pulse significantly improves the signal-to-noise ratio. We measure coherent phonon-induced reflectivity changes with laser pulses shorter than 50 fs derived from a Kerr-lens mode-locked Ti:sapphire laser with a repetition rate of $82 \mathrm{MHz}$. The sample is $n$-doped, $3 \times 10^{17}-\mathrm{cm}^{-3}$ GaAs wafers with (100) orientation. The surface field due to band bending originating from the Fermi-level pinning ${ }^{11}$ and the width of depletion layer estimated from the Schottky model $^{12}$ are $\sim 200 \mathrm{kV} / \mathrm{cm}$ and $\sim 50 \mathrm{~nm}$, respectively. The absorption length at $1.47 \mathrm{eV}, 1.0 \mu \mathrm{m},{ }^{13}$ for our present experiments is much smaller than the width of the depletion layer. This satisfies a nearly homogeneous distribution of the optically excited carrier density in the depletion layer. All measurements are performed at room temperature. At normal incidence on a (100)-oriented sample, the LEO effect leads to anisotropic reflectivity changes, ${ }^{14}$ whereas the NEO effect 


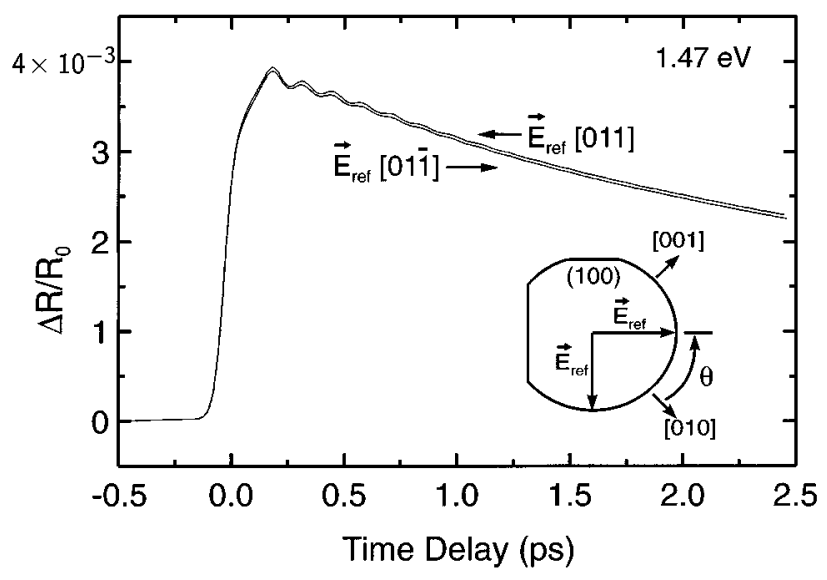

FIG. 1. Transient reflectivity changes of a (100)-oriented $n$-doped $\left(3 \times 10^{17} \mathrm{~cm}^{-3}\right)$ GaAs. The excitation density is about $10^{18} \mathrm{~cm}^{-3}$. The upper and lower traces correspond to reflectivity changes with the polarization parallel to the [011] and [01] crystal axes, respectively. The inset shows the polarization components $\left( \pm \mathbf{E}_{\text {ref }}\right)$ of the probe beam with respect to the crystal axes of a (100) wafer.

leads to an isotropic change. ${ }^{15}$ For a (110)-oriented sample, only the NEO contributions can be observed. To separate the isotropic from the anisotropic reflectivity changes at a (100) surface, the reflected beam is split into its polarization components, parallel to the $[011]$ and $[01 \overline{1}]$ crystal axes, respectively. This measurement scheme allows an exact comparison of the contributions of the different polarizations without any change of the spot position or the overlap of pump and probe on the sample.

The time-resolved reflectivity signature of (100) GaAs excited with a laser pulse with a photon energy of $1.47 \mathrm{eV}$ is shown in Fig. 1 for two different polarizations of the probing laser pulse. At an optically excited carrier density of $10^{18}$ $\mathrm{cm}^{-3}$ they differ only slightly at the scale of $\Delta R / R_{0} \sim 10^{-3}$. The main feature results from the saturation, the interband transition, and the electric-field-induced changes of the absorption. The corresponding refractive index changes are isotropic. The field-induced absorption changes result from the ultrafast screening of the surface depletion field by photoexcited carriers. These field changes lead to a reflectivity change due to the Franz-Keldysh effect as has been shown in time-resolved transmission experiments on the field dynamics in a GaAs modulator structure. ${ }^{16}$ The periodic $\mathrm{THz}$ modulation superimposed on the reflectivity signature arises from a coherent LO-phonon-plasmon mode that is excited by the impulsive screening of the surface electric field of GaAs. The frequency of the modulation is $7.6 \mathrm{THz}$. This frequency corresponds to the frequency of the lower branch of the LO-phonon-plasmon-coupled modes. ${ }^{8}$ At the high densities used in our experiments, the upper branch cannot be observed due to the limited time resolution. At low excitation densities beating phenomena are observed indicating that both phonon-plasmon branches are excited. The same effect has been observed in $2-\mathrm{eV}$ excitation experiments, ${ }^{17}$ where the phonon-plasmon modes were probed using the LEO effect only. For the interpretation of the plasmon-phonon-coupled mode, one has to consider that we excite a nonequilibrium carrier distribution that is strongly inhomogeneous due to the spatial variation of the intensity over the focus of the pump beam. ${ }^{18,19}$ However, at high densities the frequency of the lower phonon-plasmon mode approximately equals the TO frequency $(8.05 \mathrm{THz}$, for $q=0$ ) over a large range of densities. Hence, the spatial inhomogeneity hardly leads to a smearing out of the oscillations of the lower phonon-plasmon mode. For a further reduction of the smearing-out effect, we made the focus of the probe about three times smaller than that of the pump. The dephasing times observed are in the subpicosecond regime, which is significantly shorter than that of the bare LO phonon $(3.5-4.0 \mathrm{ps})$ observed at low excitation densities ${ }^{2}$ in accordance with time-resolved Raman experiments. ${ }^{20}$ This difference originates partly still from the variation of the carrier density at the probe spot. This leads to an inhomogeneous broadening of observed modes and this broadening is great if one compares it with the homogeneous linewidth of a bare LO phonon. More relevant, the coupled nonequilibrium plasmon is damped more strongly than the phonon eigenmode due to carrier-carrier scattering. In the same density range, the thermalization of nonequilibrium electrons has been determined to be $200 \mathrm{fs}$ at room temperature and at the same photon energy. ${ }^{21}$ However, the dephasing time of the coupled phonon-plasmon oscillations is found to be about three times longer than the thermalization time of 200 fs. We attribute this result to the phononlike part of the coupled mode, which does not lose its coherence even in the presence of much faster momentum scattering rates within the carrier plasma. Increased linewidths of phonon-plasmoncoupled modes have also been found in Raman scattering experiments. ${ }^{22}$ Most recently, the electroabsorption due to bare plasmon oscillation has been observed in fs transmission experiments in the GaAs diode structure with built-in field. ${ }^{23}$ There, dephasing times $<400$ fs have been observed at a lattice temperature of $90 \mathrm{~K}$ and carrier densities of $10^{16} \mathrm{~cm}^{-3}$. These dephasing times are significantly shorter than the ones we observe at room temperature, higher densities, inhomogeneous carrier, and field distributions in a doped sample. This astonishing difference confirms our interpretations of the stability of the phonon-plasmon-coupled mode. Detailed investigations on the plasmon-phonon coupling and the plasmon dephasing for near-band-gap excitation will be published in a forthcoming paper. For further interpretation of our experiments, we note that the conclusions we draw do not depend on whether we observe the unscreened LO phonon at low densities or phonon-plasmoncoupled modes at higher excitation densities, since both are longitudinal modes associated with a macroscopic electric field.

For a detailed analysis of the polarization selection rules of the detection mechanism, the oscillations extracted from Fig. 1 are shown enlarged at a scale of $\Delta R / R_{0} \sim 10^{-5}$ in Fig. 2. At this scale, noticeable differences between the two probe-beam polarizations are observed. The amplitude of the reflectivity changes for polarization along the [011] direction is larger than that for the polarization along the [011] direction. In the inset of Fig. 2 the difference between these two polarizations is plotted at a scale of $\Delta R / R_{0} \sim 10^{-6}$. This difference corresponds directly to changes in the refractive index $\Delta n$ determined by the first-order Raman tensor of Eq. 


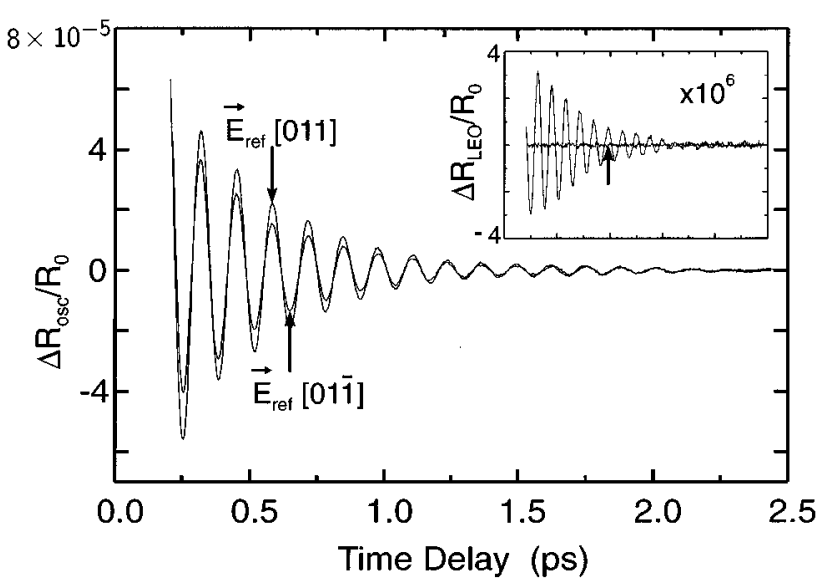

FIG. 2. Oscillatory parts extracted from the reflectivity changes of Fig. 1. The inset shows the oscillations obtained by directly measuring the anisotropic LEO effect by subtraction of the signal with polarization along [011] from that with polarization along $[01 \overline{1}]$. The curve indicated by an arrow is measured with the sample rotated by $45^{\circ}$.

(1). For the LO-phonon mode, for which $Q$ is linearly related to $F$, these changes can be equivalently expressed by the LEO effect:

$$
\Delta n_{\mathrm{LEO}} \sim n_{0}^{3}\left(r_{e}+r_{i}\right) F \sin (2 \theta),
$$

where $n_{0}$ denotes the unperturbed refractive index, $r_{e}$ and $r_{i}$ are the electronic and the ionic contributions to the linear electro-optic coefficient $r_{41}\left(=r_{e}+r_{i}\right),{ }^{24}$ and $F$ is the change of the surface depletion field including the macroscopic LOphonon field. $\theta$ is the azimuth angle between the polarization component of the reflected probe beam and the [010] crystal axis (see inset of Fig. 1). According to the symmetry of the LEO tensor, this contribution disappears after rotating the sample by $45^{\circ}$ as shown in the inset of Fig. 2 (curve marked by arrow). This demonstrates that the anisotropic LEO contribution is smaller than the isotropic modulation by one order of magnitude.

By tuning the central wavelength of the laser pulse, we measure the dispersion of both the LEO and the NEO effect. The excitation density of the photoexcited carriers is kept constant at $10^{18} \mathrm{~cm}^{-3}$ in order to achieve the same coherentphonon amplitude at all photon energies. Figure 3 shows the dispersion of the LEO and the NEO effect as derived from the photon-energy dependence of the oscillation amplitude. The strong enhancement of the NEO effect in comparison to the LEO effect in the near-band-gap regime as derived from coherent-phonon amplitudes is clearly observed. It agrees well with the results of an earlier study on a Mach-Zehnder phase modulator. ${ }^{25}$ The observed dispersion follows the general dispersion of the Franz-Keldysh effect. However, distinct Franz-Keldysh oscillations in the initial phonon amplitude cannot be observed due to the field inhomogeneities at the surface and the broad energy width of the laser pulse. The fact that the anisotropic contribution depends only weakly on the photon energy above the band gap indicates that neither the LEO effect nor the phonon-generation mechanism shows a significant dispersion above the band gap. Thus, the strong enhancement of the isotropic NEO con-

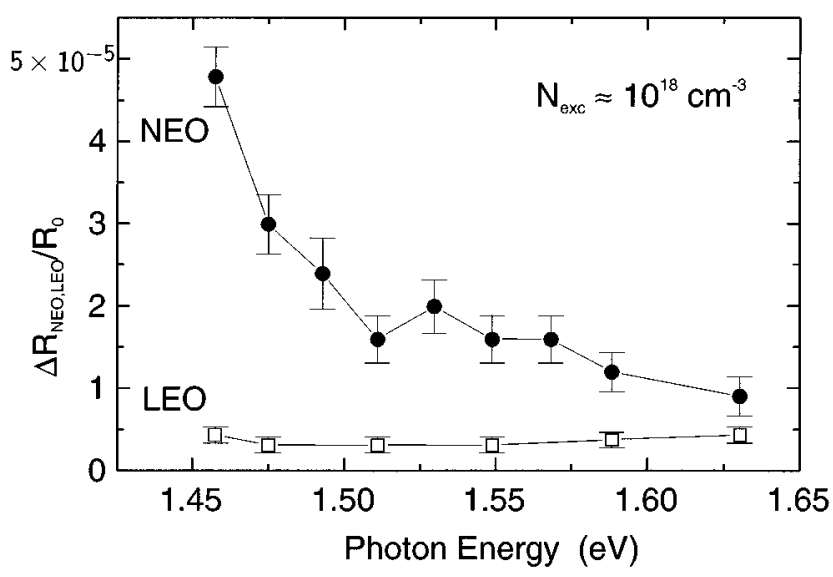

FIG. 3. The amplitude of the LEO (open squares) and NEO (solid circles) oscillation amplitude vs the photon energy of the laser pulses extracted at a delay time of $380 \mathrm{fs}$.

tribution near the band gap is solely due to the resonant character of the nonlinear electro-optic detection mechanism.

Recently, it has been proposed that the coherent excitation of a direct and a phonon-assisted interband transition can lead to a modulation of the interband oscillator strength with the longitudinal optical phonon frequency. ${ }^{26}$ However, this quantum beat effect cannot be responsible for our observations, since the interband dephasing time is extremely short (on the order of $100 \mathrm{fs}$ ) at room temperature. In addition, the required excitonic effect is strongly suppressed due to surface field-induced ionization and the Coulomb screening at these carrier densities.

The electro-optic changes of the reflectivity can be expressed as the real part of the nonlinear optical susceptibility. Taking into account the unscreened portion of the inhomogeneous surface depletion field $\left(F_{s}\right)$, the electric field can be written as $F_{s}+F_{0} e^{-t / \tau} \cos (\Omega t)$, where $F_{0}$ is the amplitude of the longitudinal field produced by the phonons, $\Omega$ the oscillation frequency, and $\tau$ the dephasing time of the oscillation. The unscreened background field in the vicinity of the surface during the transport duration of the nonequilibrium carriers is much larger than the longitudinal field $\left(F_{0}\right)$ produced by the phonons. ${ }^{7}$ Hence, the quadratic electro-optic effect proportional to $F_{s} F_{0}(\Omega)$ is larger than that proportional to $F_{0}^{2}(2 \Omega)$. Due to the permutation of the frequency arguments in $\chi^{(n)}$, higher order in $F_{0}(\Omega)$ leads to higher harmonics as well as the fundamental term of $\Omega$. In the present case, we observe changes in the reflectivity with the phonon frequency $\Omega$. Oscillations with higher harmonics cannot be resolved due to the limited time resolution. Hence, we will consider only terms with the fundamental frequency $\Omega$ in the expansion of the nonlinear susceptibility:

$$
\begin{aligned}
\Delta n^{\mathrm{NL}}(\Omega) \sim & \chi^{(2)} F_{0}(\Omega) e^{-t / \tau_{1}} \\
& +f\left(\chi^{(3)} F_{s}, \chi^{(5)} F_{s}^{3}, \ldots\right) F_{0}(\Omega) e^{-t / \tau_{1}}+\cdots .
\end{aligned}
$$

The first term describes the LEO effect, while all higherorder terms contribute to the NEO effect. In this equation it is assumed that $\chi^{(4)}$ is negligibly small compared to $\chi^{(2)}$. 
The function $f$ depends on the surface depletion field $F_{s}$ and the nonlinear optical susceptibilities $\chi^{(2 n+1)}$ that describe the Franz-Keldysh effect. ${ }^{10}$

Since the isotropic NEO signal depends on the unscreened background field, $F_{s}$, the time dependence of the total NEO signal contains the time evolution of the surface field in the presence of carrier transport. Thus, the dephasing for the NEO and the LEO response is expected to be different. These different dephasing responses are also clearly illustrated by the amplitude difference between the two polarizations in delay time in Fig 2. Figure 4 shows the dephasing of the isotropic and anisotropic oscillations extracted from Fig. 2. Each oscillation is fitted numerically and both traces are presented with a normalized amplitude at zero time delay. The short transport time associated with $F_{s}$ in the NEO signal leads to a faster decay time of $\sim 400$ fs compared to $\sim 530 \mathrm{fs}$ for the LEO signal.

In conclusion, we report on the observation of coherent phonon oscillations via the nonlinear electro-optic effect. This effect is based on the Franz-Keldysh effect, which is strongly enhanced near the band gap as compared to the linear electro-optic effect. The polarization selection rules enable the separation of the linear and the nonlinear electrooptic effects. Our findings show that the NEO effect is very important in the interpretation of time-resolved data measured in the presence of electric fields near the band gap. Similar strong nonlinear electro-optic effects are expected in quantum-well structures due to the quantum confined Stark

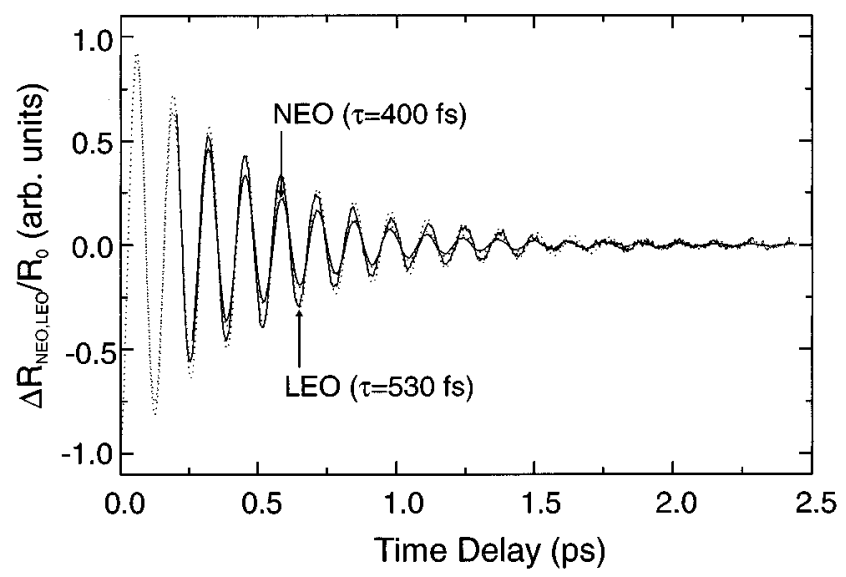

FIG. 4. Time dependence of the anisotropic and isotropic oscillations normalized at zero time delay (solid lines). The dephasing time constants $\tau$ are obtained from numerical fits (dotted lines).

effect $^{27}$ or the Wannier-Stark effect. They might open the way to use these structures as $\mathrm{THz}$ electro-optic oscillators close to the optical phonon frequencies.

We thank S. Hunsche for fruitful discussions, and A.M.T. Kim and K. Wienecke for technical assistance. This work was supported by the Alfried-Krupp Stiftung and the Deutsche Forschungsgemeinschaft (Grant No. KU 540/15-1).
${ }^{1}$ S. DeSilvestri, J.G. Fujimoto, E.B. Gamble, L.R. Williams, and K.A. Nelson, Chem. Phys. Lett. 116, 146 (1985).

${ }^{2}$ G.C. Cho, W. Kütt, and H. Kurz, Phys. Rev. Lett. 65, 764 (1990).

${ }^{3}$ T.K. Cheng, J. Vidal, H.J. Zeiger, G. Dresselhaus, M.S. Dresselhaus, and E.P. Ippen, Appl. Phys. Lett. 59, 1923 (1991).

${ }^{4}$ J.M. Chwalek, C. Uher, J.F. Whitaker, and G.A. Mourou, Appl. Phys. Lett. 58, 980 (1991).

${ }^{5}$ H. Roskos, M.C. Nuss, J. Shah, K. Leo, D.A.B. Miller, A.M. Fox, S. Schmitt-Rink, and K. Köhler, Phys. Rev. Lett. 68, 2216 (1992); T. Dekorsy, P. Leisching, K. Köhler, and H. Kurz, Phys. Rev. B 50, 8106 (1994).

${ }^{6}$ H.J. Zeiger, J. Vidal, T.K. Cheng, E.P. Ippen, G. Dresselhaus, and M.S. Dresselhaus, Phys. Rev. B 45, 768 (1992).

${ }^{7}$ T. Dekorsy, T. Pfeifer, W. Kütt, and H. Kurz, Phys. Rev. B 47, 3842 (1993).

${ }^{8}$ A. Pinczuk and E. Burstein, in Light Scattering in Solids, edited by M. Cardona (Springer-Verlag, Berlin, 1975).

${ }^{9}$ M. Cardona, in Light Scattering in Solids II, edited by M. Cardona and G. Güntherodt (Springer-Verlag, Berlin, 1982).

${ }^{10}$ D.E. Aspnes, Surf. Sci. 37, 418 (1973).

${ }^{11}$ H. Hasegawa, H. Ishii, T. Sawada, T. Saitoh, S. Konishi, Y. Liu, and H. Ohno, J. Vac. Sci. Technol. B 6, 1184 (1988).

${ }^{12}$ S.M. Sze, Physics of Semiconductor Devices (Wiley, New York, 1981).

${ }^{13}$ Handbook of Optical Constants of Solids, edited by E.D. Palik (Academic, London, 1985).

${ }^{14}$ A. Yariv, Quantum Electronics (Wiley, New York, 1988).

${ }^{15}$ Y.R. Shen, The Principles of Nonlinear Optics (Wiley, New York, 1984): for example, the polarization selection rule for $\chi^{(3)}$.
${ }^{16}$ H. Heesel, S. Hunsche, H. Mikkelsen, T. Dekorsy, K. Leo, and H. Kurz, Phys. Rev. B 47, 16000 (1993).

${ }^{17}$ W.A. Kütt, W. Albrecht, and H. Kurz, IEEE J. Quantum Electron. 28, 2434 (1992).

${ }^{18}$ C.L. Collins and P.Y. Yu, Solid State Commun. 51, 123 (1984).

${ }^{19}$ A.V. Kuznetsov and C.J. Stanton, Phys. Rev. B 51, 7555 (1995).

${ }^{20}$ A. Kash, S.S. Jha, and J.C. Tsang, Phys. Rev. Lett. 58, 1869 (1987); F. Vallee, Phys. Rev. B 49, 2460 (1994).

${ }^{21}$ S. Hunsche, H. Heesel, A. Ewertz, H. Kurz, and J.H. Collet, Phys. Rev. B 48, 17818 (1993).

${ }^{22}$ G. Abstreiter, R. Trommer, M. Cardona, and A. Pinczuk, Solid State Commun. 30, 703 (1979).

${ }^{23}$ W. Sha, A. Smirl, and W.F. Tseng, Phys. Rev. Lett. 74, 4273 (1995).

${ }^{24}$ Due to the fact that GaAs is polar and lacks inversion symmetry, the field-induced changes of the optical susceptibility in the case of LO phonon arise from both $(\partial \chi / \partial Q)_{F}\left(\sim r_{i}\right)$ and $(\partial \chi / \partial F)_{Q}$ $\left(\sim r_{e}\right)$ in the linear case where $\chi^{(2)} \sim r_{41}=r_{i}+r_{e}$ is valid. However, the nonlinear electro-optic effect, which does not require a lack of inversion symmetry, is dominated by the electronic contribution. See for the description of the linear electro-optic coefficient C.-C. Shih and A. Yariv, J. Phys. C 15, 825 (1982); I.P. Kaminow and E.H. Turner, Phys. Rev. B 5, 1564 (1972).

${ }^{25}$ T.E. Van Eck, L.M. Walpita, W.S.C. Chang, and H.H. Wieder, Appl. Phys. Lett. 48, 451 (1986).

${ }^{26}$ D.B. Tran Thoai and H. Haug, Phys. Rev. B 47, 3574 (1993).

${ }^{27}$ D.A.B. Miller, D.S. Chemla, T.C. Damen, T.H. Wood, C.A. Burrus, Jr., A.C. Gossard, and W. Wiegmann, IEEE J. Quantum Electron. 21, 1462 (1985). 